\title{
Preditores e Incidência de Complicações Vasculares Após a Realização de Intervenções Coronárias Percutâneas: Achados do Registro IC-FUC
}

\author{
Leonardo G. Zanatta ${ }^{1}$, Cristiano O. Cardosoํ, Fábio M. Mota ${ }^{1}$, Elias P. Conti ${ }^{1}$, Dayane Diehl' ${ }^{1}$, \\ Ana Paula R. Rodrigues ${ }^{1}$, Fernanda O. Camozzatto', La Hore Rodrigues Jr. ${ }^{1}$, \\ Carlos A. M. Gottschall1', Rogério Sarmento-Leite', Alexandre Schaan de Quadros ${ }^{1}$
}

\section{RESUMO}

Introdução: As complicações vasculares (CV) são uma das principais causas de morbidade e mortalidade em pacientes submetidos a intervenções coronárias percutâneas (ICPs), porém estudos anteriores não refletem a prática atual. Objetivos: Avaliar a incidência de CV e seus preditores, em uma população de pacientes tratada com ICP contemporaneamente. Método: Estudo observacional de corte transversal, com implantes de stents coronários, de janeiro de 2000 a dezembro de 2007. As características clínicas e angiográficas e a evolução intra-hospitalar foram avaliadas e registradas em banco de dados informatizado. Foram excluídos aqueles com óbito hospitalar ou cirurgia cardíaca de urgência. CV foram definidas como sangramento maior, cirurgia vascular ou hematoma $>10 \mathrm{~cm}$. Os dados foram analisados com SPSS 11.0, e as características dos pacientes com e sem CV foram comparadas com teste $t$ de Student e teste do qui-quadrado. Os preditores independentes de CV foram identificados por análise de regressão logística múltipla. Resultados: Total de 4.595 pacientes com 5.485 stents implantados, com média de idade de $60,64 \pm 10,65$ anos e $32 \%$ de mulheres. As ICPs foram realizadas pela via femoral em $95 \%$ dos casos e pela via radial em $5 \%$, e em $85 \%$ dos procedimentos foram utilizados introdutores $6 \mathrm{~F}$ (em $15 \%$ foram usados introdutores 7F). Foram registradas CV em 162 (3,3\%) pacientes. Por análise multivariada, o único preditor de $\mathrm{CV}$ foi o uso de introdutores $7 \mathrm{~F}$ (razão de chance $=3,05$, intervalo de confiança $=1,2-7,8 ; p=0,02$ ). Pelo teste de Hosmer-Lemeshow-goodness-of-fit, o modelo utilizado demonstrou boa calibração para a amostra analisada (quiquadrado $=6,9 ; p=0,55)$. Conclusão: A prevalência de
SUMMARY

Predictors and Incidence of Vascular
Complications After Percutaneous Coronary
Interventions: Findings from the IC-FUC Registry

Background: Vascular complications (VC) following percutaneous coronary intervention $(\mathrm{PCl})$ are an important cause of morbidity and mortality. However, available data do not reflect current interventional cardiology practice. Objective: To determinate the incidence of $\mathrm{VC}$ and its predictors in a population treated with $\mathrm{PCl}$ in contemporary practice. Methods: Cross-sectional study with coronary stent implantation conducted from January/2000 to December/2007. Clinical and angiographic characteristics, as well as in-hospital evolution, were evaluated and recorded in a database. Exclusion criteria included in-hospital death and urgent heart surgery. VC were defined as major bleeding, vascular surgery or hematoma $>10 \mathrm{~cm}$. Data were analyzed using SPSS 11.0 and the characteristics of patients with and without VC were compared using the Student's $t$ test and chi-square test. Multiple Logistic Regression Analysis was performed to determinate the independent predictors of VC. Results: A total of 4,595 patients with 5,485 stents were included in this analysis. Mean age was $60.64 \pm 10.65$ years and $32 \%$ of the patients were female. The transfemoral approach was used in $95 \%$ of the $\mathrm{PCls}$ and the transradial approach in 5\%. Six French and 7 French introducers were used in $85 \%$ and $15 \%$ of the PCls, respectively. A total of $162(3.3 \%)$ patients experienced VC. The multivariate analysis determined that the only predictor for VC was the use of 7 French introducers (odds ratio $=3.05,95 \%$ confidence interval $=1.2-7.8 ; p=0.02$ ).

1 Instituto de Cardiologia do Rio Grande do Sul/Fundação Universitária de Cardiologia - Porto Alegre, RS.

Correspondência: Alexandre Quadros. Unidade de Pesquisa do IC/ FUC - Av. Princesa Isabel, 370 - Santana - Porto Alegre, RS CEP 90620-001 - Fax: (51) 3219-2802/ramais 22-23-24

E-mail: aquadros@cardiol.br

Recebido em: 31/7/2008 • Aceito em: 4/9/2008 

Achados do Registro IC-FUC. Rev Bras Cardiol Invas. 2008;16(3):301-306.

CV maiores foi baixa, tendo como único preditor o calibre do introdutor arterial utilizado.

DESCRITORES: Angioplastia coronária transluminal percutânea/efeitos adversos. Cateterismo cardíaco/efeitos adversos. Doenças vasculares/etiologia. Fatores de risco.

D esde a introdução dos stents no campo da Cardiologia Intervencionista, os resultados imediatos das intervenções coronárias percutâneas (ICPs) modificaram-se. Ocorreram redução substancial das taxas de oclusão aguda se comparada à era da angioplastia com balão e melhora do sucesso técnico resultante da evolução dos materiais ${ }^{1,2}$. Embora a taxa de sucesso agudo tenha aumentado, o uso freqüente de dupla terapia antiplaquetária, inibidores de glicoproteína Ilb/IIla e regimes de anticoagulação mais agressivos promoveram incremento em complicações vasculares ${ }^{3}$.

No contexto das ICP, as complicações vasculares são manifestações relativamente freqüentes, podendo sua incidência oscilar entre 1\% e 14\%. Adicionalmente, esse tipo de complicação tem sido considerado uma das principais causas de morbidade e mortalidade nesses pacientes ${ }^{4}$. Tem-se buscado, com o desenvolvimento de novos dispositivos, minimizar a injúria ao leito vascular arterial, determinando, conseqüentemente, redução significativa na prevalência dessas complicações. Hematoma local, retroperitoneal, pseudo-aneurisma e formação de fístula arteriovenosa determinam aumento do tempo de permanência hospitalar, custos diretos relacionados ao procedimento, desconforto e riscos adicionais aos pacientes em decorrência de possíveis procedimentos de reparo cirúrgico, compressão mecânica e transfusões sanguíneas ${ }^{5-10}$.

Alguns dos principais preditores de complicações vasculares descritos são: sexo feminino, idade superior a 70 anos, tempo de coagulação ativada > 175 segundos na retirada do introdutor arterial, heparina de baixo peso molecular, clopidogrel antes do procedimento, diabetes melito, hipertensão arterial sistêmica, índice de massa corporal > $25 \mathrm{~kg} / \mathrm{m}^{2}$ e surgimento de hematoma durante o procedimento ${ }^{11,12}$. No entanto, esses dados refletem a realidade de outros países, e muitos estudos já não representam a prática médica contemporânea. Diante disso, objetivamos avaliar complicações vasculares e seus possíveis preditores em pacientes tratados com ICP em um centro de referência.

\section{MÉTODO}

\section{Pacientes}

A partir de janeiro de 2000 incluímos, prospectiva e consecutivamente, no Registro IC-FUC, todos os
By the Hosmer-Lemeshow-goodness-of-fit test, the model used demonstrated good calibration for sample analyzed (chi-square $=6.9 ; p=0.55)$. Conclusion: The prevalence of VC was low and its only predictor was the diameter of the arterial introducer.

DESCRIPTORS: Percutaneous transluminal coronary angiography/adverse effects. Heart catheterization/adverse effects. Vascular diseases/etiology. Risk factors.

pacientes submetidos a ICP para tratamento de cardiopatia isquêmica tratados no Instituto de Cardiologia do Rio Grande do Sul/Fundação Universitária de Cardiologia, em Porto Alegre, RS. Para fins dessa análise, incluímos os 4.595 pacientes tratados até dezembro de 2007. Variáveis relacionadas aos fatores de risco para doença cardiovascular, indicação do procedimento, detalhes técnicos da intervenção, complicações e seguimento intra-hospitalar foram prospectivamente registrados em um formulário específico e digitados em um banco de dados dedicado. Todos os pacientes submetidos a ICP foram selecionados, tendo sido excluídos somente aqueles que apresentaram óbito hospitalar não relacionado à complicação vascular ou que foram submetidos a cirurgia cardíaca de urgência. Os pacientes foram acompanhados durante o período de internação, sendo os eventos registrados de maneira prospectiva. O estudo foi aprovado pelo comitê de ética e pesquisa institucional, e todos os pacientes assinaram um termo de consentimento informado.

\section{Intervenção coronária percutânea}

As ICPs foram realizadas de acordo com as técnicas habituais e conforme orientações das diretrizes vigentes ${ }^{13-15}$. Em resumo, os procedimentos foram realizados por via femoral ou radial, sendo a artéria coronária cateterizada com cateter apropriado. Pré-dilatação foi utilizada a critério do operador, bem como o uso de inibidores de glicoproteína Ilb/Illa. Todos os pacientes receberam heparina não-fracionada no início do procedimento (70-100 U/kg), com a administração de doses adicionais, se necessário, para manter o tempo de coagulação ativada (TCA) entre 250 e 350 segundos. Dupla terapia antiplaquetária foi utilizada em todos os pacientes (ácido acetilsalicílico [AAS] $200 \mathrm{mg} /$ dia e clopidogrel 300-600 mg em dose de ataque e manutenção de $75 \mathrm{mg} /$ dia ou ticlopidina 250 mg a cada doze horas). A terapia antiplaquetária dupla foi mantida por trinta dias em pacientes tratados com stents convencionais e por seis meses naqueles que receberam stent farmacológico. Os introdutores utilizados nas abordagens radiais foram retirados na própria sala de hemodinâmica, independentemente dos níveis de TCA. Os introdutores femorais foram retirados quatro horas após o início da dose de heparina. A técnica de compressão femoral manual foi utilizada em todos os pacientes tratados por essa via. 


\section{Complicações vasculares}

Complicações vasculares foram definidas como: (1) presença de hematoma $>10 \mathrm{~cm}$ no local da punção arterial; (2) sangramento maior, caracterizado como queda de hemoglobina $>3 \mathrm{~g} / \mathrm{dl}$ ou necessidade de transfusão de concentrado de hemácias; e (3) necessidade de correção cirúrgica da complicação (hematoma retroperitoneal, pseudo-aneurisma ou formação de fístula arteriovenosa).

\section{Análise estatística}

As variáveis são apresentadas em porcentual e média \pm desvio padrão. Para comparação entre os grupos com e sem complicações vasculares, foram utilizados os testes de qui-quadrado e $t$ de Student quando comparadas variáveis categóricas e contínuas, respectivamente. Regressão logística múltipla foi aplicada para identificar possíveis preditores de complicações vasculares. Foram utilizadas, nesse modelo, variáveis com significância estatística $(p<0,05)$ na análise univariada ou sem significância estatística, mas com relevância biológica. O teste de Hosmer-Lemeshow goodness-of-fit foi utilizado para avaliar a calibração dos modelos. Valor de $p$ bicaudal $<0,05$ foi considerado estatisticamente significativo para os testes aplicados. Todos os dados foram analisados por meio do software SPSS para Windows versão 11.0. Para fins de pesquisa, as complicações foram consideradas em análise combinada.

\section{RESULTADOS}

No período do estudo, 4.595 pacientes foram submetidos a ICP e 5.485 stents foram implantados. As características demográficas dos pacientes encontram- se descritas na Tabela 1. Podemos observar que a maioria dos pacientes era do sexo masculino (68\%) e com média de idade de 60,64 $\pm 10,65$ anos. Houve predomínio de hipertensão arterial sistêmica (77\%) e infarto agudo do miocárdio (21\%) no grupo de pacientes que não apresentou complicações vasculares. Não houve diferença entre os desfechos em relação a diabetes melito e tabagismo. A apresentação clínica foi, na sua maioria, de síndromes coronárias agudas (57\%), sendo o uso de inibidores de glicoproteína IIb/IIla inferior a $5 \%$.

As características relacionadas ao procedimento estão apresentadas na Tabela 2. Os pacientes sem complicações vasculares apresentaram índices de sucesso no procedimento superiores $(98,4 \%)$ aos daqueles com complicações $(92,1 \%)$ ( $p<0,001)$. Introdutor $6 \mathrm{~F}$ foi utilizado em $85 \%$ dos casos, em $15 \%$ foram utilizados introdutores 7F, e a via radial foi usada em aproximadamente $5 \%$. As intervenções realizadas por via radial apresentaram índices de complicações vasculares significativamente menores que aquelas realizadas pela via femoral $(0,6 \%$ vs. $3,4 \% ; p=0,008)$. Disfunção ventricular e angioplastia primária não se mostraram preditores de complicações vasculares nesta análise.

No total, foram constatados $162(3,3 \%)$ casos de complicações vasculares, considerados como desfecho composto (hematoma, necessidade de transfusão, pseudo-aneurisma e fístula arteriovenosa). Na Tabela 3, diversas variáveis clínicas e relacionadas ao procedimento foram consideradas como potenciais preditores de complicações vasculares, e avaliadas por análise de regressão logística. Apenas o calibre do introdutor alcançou significância estatística (razão de chance $=3,05$; intervalo de confiança de

TABELA 1

Características clínicas dos pacientes

\begin{tabular}{lcccc}
\hline Características & Geral $(\mathbf{n}=\mathbf{4 . 5 9 5})$ & CV presente $(\mathbf{n}=\mathbf{1 6 2})$ & CV ausente $(\mathbf{n}=\mathbf{4 . 4 3 3})$ & \\
\hline Demográficas & & & & $\mathbf{p}^{*}$ \\
Idade, anos & $60,64 \pm 10,65$ & $60,8 \pm 9,8$ & $60,4 \pm 10,7$ & 0,7 \\
Mulheres, \% & 32 & 33 & 31 & 0,6 \\
Peso, kg & $76,04 \pm 13,86$ & $73,96 \pm 12,74$ & $76,42 \pm 14,05$ & 0,34 \\
HAS, \% & 76 & 59 & 47,3 & 0,01 \\
Tabagismo, \% & 47,3 & 45,9 & 16 & 0,8 \\
DM, \% & 20 & 20 & 21 & 0,6 \\
IAM prévio, \% & 20 & 10 & 42,5 & $\mathrm{~N}$ \\
Quadro clínico, \% & & & 47,9 & $\mathrm{NS}$ \\
Angina estável & 42,5 & 42,8 & 10,1 & $\mathrm{NS}$ \\
Angina instável & 47,1 & 47,9 & 9,6 & $\mathrm{NS}$ \\
IAM & 10,1 & 9,6 & & \\
\hline
\end{tabular}

* Refere-se à comparação entre pacientes com e sem complicações vasculares.

$\mathrm{CV}=$ complicação vascular; $\mathrm{DM}$ = diabetes melito; HAS = hipertensão arterial sistêmica; IAM = infarto agudo do miocárdio; $\mathrm{n}=$ número de pacientes; NS = não-significante. 
TABELA 2

Características angiográficas dos pacientes conforme a ocorrência de complicações vasculares

\begin{tabular}{lcccc} 
Características & Geral $(\mathbf{n}=\mathbf{4 . 5 9 5})$ & CV presente $(\mathbf{n}=\mathbf{1 6 2})$ & CV ausente $(\mathbf{n}=\mathbf{4 . 4 3 3})$ & $\mathbf{p}^{*}$ \\
\hline Uniarterial, \% & 28 & 39 & 27 & 0,002 \\
Disfunção do VE & 9,5 & 10,6 & 9,4 & 0,67 \\
Sucesso, \% & 98,1 & 92,1 & 98,4 & $<0,001$ \\
Primeira ACTP, \% & 9,6 & 10 & 10 & 0,97 \\
7 French, \% & 15 & 22 & 14,9 & 0,22 \\
Radial, \% & 5,3 & 0,6 & 5,5 & 0,008
\end{tabular}

* Refere-se à comparação entre pacientes com e sem complicações vasculares.

ACTP = angioplastia coronária transluminal percutânea; $C V=$ complicação vascular; VE = ventrículo esquerdo; $\mathrm{n}=$ número de pacientes.

TABELA 3

Análise multivariada para os preditores de complicação vascular

\begin{tabular}{lccc} 
Variável & Razão de chance & IC $\mathbf{9 5} \%$ & Valor de $\mathbf{p}$ \\
\hline Sucesso & 0,01 & $0,00-3,75$ & 0,93 \\
IAM prévio & 0,82 & $0,34-1,99$ & 0,66 \\
Introdutor & 3,05 & $1,20-7,80$ & 0,02 \\
Via & 0,42 & $0,05-3,06$ & 0,43 \\
HAS & 0,57 & $0,24-1,35$ & 0,20 \\
Peso & 0,99 & $0,95-1,02$ & 0,48 \\
Idade & 1,01 & $0,97-1,05$ & 0,57 \\
Sexo & 0,878 & $0,35-2,23$ & 0,79 \\
\hline
\end{tabular}

IAM = infarto agudo do miocárdio; IC = intervalo de confiança; HAS = hipertensão arterial sistêmica.

95\% [IC 95\%] = 1,2-7,8; p = 0,02), demonstrando relação direta entre calibre do introdutor e incremento em complicações vasculares. Pelo teste de HosmerLemeshow goodness-of-fit, o modelo utilizado demonstrou boa calibração para a amostra analisada (quiquadrado $=6,9 ; p=0,55)$.

\section{DISCUSSÃO}

Neste estudo observacional, realizado em um centro de referência para Cardiologia Intervencionista, constatamos que a taxa de complicações vasculares intrahospitalares foi de $3,3 \%$, que é relativamente baixa quando comparada a resultados previamente publicados ${ }^{16-18}$.

Por meio de análise multivariada, identificamos a relação significativa do calibre dos introdutores $7 \mathrm{~F}$ como preditor de complicações vasculares (hematoma, sangramento maior e correção cirúrgica) em relação aos dispositivos 6F, diferentemente dos resultados de estudo previamente publicado, em que o diâmetro dos introdutores não agregou maiores índices de complicações ${ }^{12}$. Em publicação recente, Brito Jr. et al. ${ }^{19}$ constataram que os preditores de complicações vasculares foram sexo feminino (razão de chance $=5,61$; IC 95\% $=1,99-15,76)$ e uso associado de inibidores de glicoproteína IIb/IIla - abciximab (razão de chance $=3,02$; IC 95\% = 1,10-8,26). Nesse mesmo estudo, houve relação significativa dos dispositivos hemostáticos de oclusão, sendo esse fator identificado como protetor em relação a complicações vasculares (razão de chance =0,36; IC 95\%=0,12-0,99). Embora o estudo de Brito Jr. et al. ${ }^{19}$ também espelhe uma amostra de procedimentos representativos em termos nacionais, ambos os trabalhos apresentam diferenças importantes que podem justificar os resultados citados. A principal diferença foi o uso de inibidores de glicoproteína Ilb/IIla muito freqüente no estudo de Brito Jr. et al. ${ }^{19}$ e muito infreqüente neste relato. Da mesma forma, os dispositivos de oclusão femoral foram usados freqüentemente no estudo citado, e não foram usados em nosso trabalho.

Recentemente, Piper et al. ${ }^{18}$ realizaram um estudo com delineamento metodológico semelhante ao do presente trabalho, analisando os preditores e as complicações vasculares em uma população de mais de 18 mil pacientes submetidos a ICP. Idade avançada (> 70 anos), sexo feminino e baixa superfície corporal foram 

Achados do Registro IC-FUC. Rev Bras Cardiol Invas. 2008;16(3):301-306.

associados a tais injúrias vasculares, porém não foram avaliadas características ligadas diretamente ao procedimento (introdutores, TCA, vias de acesso). Existem dados conflitantes em relação ao benefício do uso de dispositivos de oclusão arterial após ICP, não havendo, portanto, consenso sobre o real benefício de uso desses dispositivos quando comparados aos métodos usuais de compressão manual. Apenas a deambulação precoce é favorecida pelo uso desse tipo de dispositivo ${ }^{20-23}$.

Tanto os inibidores de glicoproteína IIb/IIla $\mathrm{a}^{3,16,24,25}$ como os agentes antitrombínicos ${ }^{26,27}$ são altamente efetivos no processo de inibição da agregação plaquetária e inativação dos fatores de coagulação, promovendo benefício significativo na redução de eventos cardiovasculares em síndromes isquêmicas agudas e infarto agudo do miocárdio. Em contrapartida, observa-se aumento da incidência de sangramento com essas estratégias. Nosso estudo não foi capaz de avaliar o impacto desse grupo de drogas como preditores de complicações vasculares, já que seu uso foi restrito a menos de $5 \%$ das intervenções.

Nos últimos anos, o emprego da técnica radial tem aumentado significativamente nos laboratórios de hemodinâmica em todo o mundo. A principal vantagem dessa técnica sobre a femoral já foi previamente demonstrada por estudos randomizados ${ }^{28}$ e meta-análises ${ }^{29}$. Se considerarmos pacientes em uso de anticoagulantes e esquemas potentes de antiagregação plaquetária, esse benefício fica ainda mais evidente ${ }^{30}$. Em nosso estudo, a técnica radial foi utilizada em apenas 5,3\% dos procedimentos, e esses pacientes tiveram taxas de complicações vasculares significativamente menores que aqueles tratados pela via femoral. No entanto, nossa análise não demonstrou essa via de acesso como preditor independente de complicações vasculares, provavelmente em virtude do número reduzido de pacientes.

\section{Limitações do estudo}

O presente estudo tem limitações que devem ser consideradas. Embora tenhamos relatado baixo índice de complicações vasculares, alguns fatores podem ter contribuído para esses achados: (1) as definições de complicações vasculares utilizadas diferem das estabelecidas nas atuais diretrizes de ICP; (2) o uso de inibidores de glicoproteína IIb/IIla foi inferior a 5\% dos procedimentos; (3) não dispomos dos dados referentes ao nível de anticoagulação (TCA) atingido durante os procedimentos ou no momento da retirada do introdutor; e (4) complicações manejadas clinicamente, como pseudo-aneurisma ou hematoma retroperitoneal, não foram incluídas na análise, somente quando realizada correção cirúrgica.

\section{CONCLUSÃO}

A conclusão principal deste estudo é que a taxa de complicações vasculares em pacientes tratados con- temporaneamente em um centro de referência para Cardiologia Intervencionista é baixa, tendo como principal preditor o calibre do introdutor arterial utilizado.

\section{REFERÊNCIAS BIBLIOGRÁFICAS}

1. Hearn JA, King SB $3^{\text {rd }}$, Douglas JS Jr, Carlin SF, Lembo NJ, Ghazzal ZM. Clinical and angiographic outcomes after coronary artery stenting for acute or threatened closure after percutaneous transluminal coronary angioplasty. Initial results with a balloon-expandable, stainless steel design. Circulation. 1993;88(5 Pt 1):2086-96.

2. Roubin GS, Cannon AD, Agrawal SK, Macander PJ, Dean LS, Baxley WA, et al. Intracoronary stenting for acute and threatened closure complicating percutaneous transluminal coronary angioplasty. Circulation. 1992;85(3):916-27.

3. Boersma E, Harrington RA, Moliterno DJ, White $H$, Théroux $\mathrm{P}$, Van de Werf $\mathrm{F}$, et al. Platelet glycoprotein Ilb/Illa inhibitors in acute coronary syndromes: a meta-analysis of all major randomised clinical trials. Lancet. 2002;359(9302):189-98.

4. Oweida SW, Roubin GS, Smith RB $3^{\text {rd }}$, Salam AA. Postcatheterization vascular complications associated with percutaneous transluminal coronary angioplasty. J Vasc Surg. 1990;12(3):310-5.

5. Lauer MA, Karweit JA, Cascade EF, Lin ND, Topol EJ. Practice patterns and outcomes of percutaneous coronary interventions in the United States: 1995 to 1997. Am J Cardiol. 2002;89(8):924-9.

6. Davis C, VanRiper S, Longstreet J, Moscucci M. Vascular complications of coronary interventions. Heart Lung. 1997; 26(2):118-27.

7. Nasser TK, Mohler ER $3^{\text {rd }}$, Wilensky RL, Hathaway DR. Peripheral vascular complications following coronary interventional procedures. Clin Cardiol. 1995;18(11):609-14.

8. Omoigui NA, Califf RM, Pieper K, Keeler G, O'Hanesian MA, Berdan LG, et al. Peripheral vascular complications in the Coronary Angioplasty Versus Excisional Atherectomy Trial (CAVEAT-I). J Am Coll Cardiol. 1995;26(4):922-30.

9. Skillman JJ, Kim D, Baim DS. Vascular complications of percutaneous femoral cardiac interventions. Incidence and operative repair. Arch Surg. 1988;123(10):1207-12.

10. Mills JL, Wiedeman JE, Robison JG, Hallett JW Jr. Minimizing mortality and morbidity from iatrogenic arterial injuries: the need for early recognition and prompt repair. J Vasc Surg. 1986;4(1):22-7.

11. Andersen K, Bregendahl M, Kaestel H, Skriver M, Ravkilde J. Haematoma after coronary angiography and percutaneous coronary intervention via the femoral artery frequency and risk factors. Eur J Cardiovasc Nurs. 2005;4(2):123-7.

12. Berry C, Kelly J, Cobbe SM, Eteiba H. Comparison of femoral bleeding complications after coronary angiography versus percutaneous coronary intervention. Am J Cardiol. 2004;94(3):361-3.

13. Silber S, Albertsson P, Avilés FF, Camici PG, Colombo A, Hamm C, et al. Guidelines for percutaneous coronary interventions. The Task Force for Percutaneous Coronary Interventions of the European Society of Cardiology. Eur Heart J. 2005;26(8):804-47.

14. Smith SC Jr, Feldman TE, Hirshfeld JW Jr, Jacobs AK, Kern MJ, King SB 3 $3^{\text {rd }}$, et al. ACC/AHA/SCAI 2005 guideline update for percutaneous coronary intervention: a report of the American College of Cardiology/American Heart Association Task Force on Practice Guidelines (ACC/AHA/SCAI Writing Committee to Update 2001 Guidelines for Percutaneous Coronary Intervention). Circulation. 2006;113(7): e166-286.

15. Mattos LA, Lemos Neto PA, Rassi A Jr, Marin-Neto JA, Sousa 
Zanatta LG, et al. Preditores e Incidência de Complicações Vasculares Após a Realização de Intervenções Coronárias Percutâneas: Achados do Registro IC-FUC. Rev Bras Cardiol Invas. 2008;16(3):301-306.

AGMR, Devito FS, et al. Diretrizes da Sociedade Brasileira de Cardiologia - Intervenção Coronária Percutânea e Métodos Adjuntos Diagnósticos em Cardiologia Intervencionista (II Edição - 2008). Rev Bras Cardiol Invas. 2008;16 Supl 2:9-88.

16. Blankenship JC, Hellkamp AS, Aguirre FV, Demko SL, Topol EJ, Califf RM. Vascular access site complications after percutaneous coronary intervention with abciximab in the Evaluation of C7E3 for the Prevention of Ischemic Complications (EPIC) trial. Am J Cardiol. 1998;81(1):36-40.

17. Kinnaird TD, Stabile E, Mintz GS, Lee CW, Canos DA, Gevorkian $\mathrm{N}$, et al. Incidence, predictors, and prognostic implications of bleeding and blood transfusion following percutaneous coronary interventions. Am J Cardiol. 2003;92(8):930-5.

18. Piper WD, Malenka DJ, Ryan TJ Jr, Shubrooks SJ Jr, O’Connor GT, Robb JF, et al. Predicting vascular complications in percutaneous coronary interventions. Am Heart J. 2003;145(6): 1022-9.

19. Brito Jr FS, Magalhães MA, Nascimento TCDC, Amorim IMG, Almeida BO, Abizaid AC, et al. Incidência e preditores contemporâneos de complicações vasculares após intervenção coronária percutânea. Rev Bras Cardiol Invas. 2007;15(4):394-9.

20. Nikolsky E, Mehran R, Halkin A, Aymong ED, Mintz GS, Lasic Z, et al. Vascular complications associated with arteriotomy closure devices in patients undergoing percutaneous coronary procedures: a meta-analysis. J Am Coll Cardiol. $2004 ; 44(6): 1200-9$.

21. Dangas G, Mehran R, Kokolis S, Feldman D, Satler LF, Pichard AD, et al. Vascular complications after percutaneous coronary interventions following hemostasis with manual compression versus arteriotomy closure devices. J Am Coll Cardiol. 2001;38(3):638-41.

22. Exaire JE, Tcheng JE, Kereiakes DJ, Kleiman NS, Applegate RJ, Moliterno DJ. Closure devices and vascular complications among percutaneous coronary intervention patients receiving enoxaparin, glycoprotein IIb/IIla inhibitors, and clopidogrel. Catheter Cardiovasc Interv. 2005;64(3):369-72.

23. Tavris DR, Gallauresi BA, Lin B, Rich SE, Shaw RE, Weintraub WS, et al. Risk of local adverse events following cardiac catheterization by hemostasis device use and gender. J Invasive Cardiol. 2004;16(9):459-64.

24. Yatskar L, Selzer F, Feit F, Cohen HA, Jacobs AK, Williams $\mathrm{DO}$, et al. Access site hematoma requiring blood transfusion predicts mortality in patients undergoing percutaneous coronary intervention: data from the National Heart, Lung, and Blood Institute Dynamic Registry. Catheter Cardiovasc Interv. 2007;69(7):961-6.

25. ESPRIT Investigators. Enhanced Suppression of the Platelet IIb/IIla Receptor with Integrilin Therapy. Novel dosing regimen of eptifibatide in planned coronary stent implantation (ESPRIT): a randomised, placebo-controlled trial. Lancet. 2000;356(9247):2037-44.

26. Nikolsky E, Mehran R, Dangas G, Fahy M, Na Y, Pocock $S$ J, et al. Development and validation of a prognostic risk score for major bleeding in patients undergoing percutaneous coronary intervention via the femoral approach. Eur Heart J. 2007;28(16):1936-45.

27. Lincoff AM, Bittl JA, Harrington RA, Feit F, Kleiman NS, Jackman JD, et al. Bivalirudin and provisional glycoprotein Ilb/IIla blockade compared with heparin and planned glycoprotein Ilb/IIla blockade during percutaneous coronary intervention: REPLACE-2 randomized trial. JAMA. 2003; 289(7):853-63.

28. Kiemeneij F, Laarman G), Odekerken D, Slagboom T, van der Wieken R. A randomized comparison of percutaneous transluminal coronary angioplasty by the radial, brachial and femoral approaches: the access study. J Am Coll Cardiol. $1997 ; 29(6): 1269-75$.

29. Agostoni P, Biondi-Zoccai GG, de Benedictis ML, Rigattieri $S$, Turri M, Anselmi M, et al. Radial versus femoral approach for percutaneous coronary diagnostic and interventional procedures: systematic overview and meta-analysis of randomized trials. J Am Coll Cardiol. 2004;44(2):349-56.

30. Choussat R, Black A, Bossi I, Fajadet J, Marco J. Vascular complications and clinical outcome after coronary angioplasty with platelet IIb/IIla receptor blockade. Comparison of transradial vs transfemoral arterial access. Eur Heart J. $2000 ; 21(8): 662-7$. 